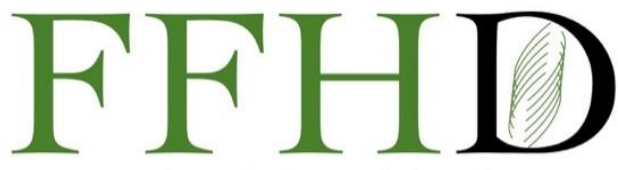

Functional Foods in Health and Disease

\title{
Continuous ascorbate infusions in the management of the patients with advanced colon cancer
}

\section{Nina Mikirova}

Riordan Clinic, 3100 North Hillside, Wichita, KS, USA.

Corresponding Author: Nina Mikirova, PhD, Director of Research, Riordan Clinic, Wichita, KS, USA.

Submission Date: July 21, 2020; Acceptance Date: September 23, 2020; Publication Date: September $30^{\text {th }}, 2020$

Please cite this article as: Mikirova, N. Continuous ascorbate infusions in the management of the patients with advanced colon cancer. Functional Foods in Health and Disease 2020. 10(9): 407-418. DOI: https://www.doi.org/10.31989/ffhd.v10i9.732

\begin{abstract}
Objective: Cytotoxic effect of ascorbic acid on colon cancer cells has been demonstrated in pre-clinical models. In this study, we analyzed data of a previous clinical trial of the treatment of late stage colon cancer patients by continuous ascorbic acid infusions.

Design: The author analyzed the effect of continuous intravenous ascorbic acid (10 g-50 g) administered by injection pumps for 6-8 weeks. Adverse effects,
\end{abstract}

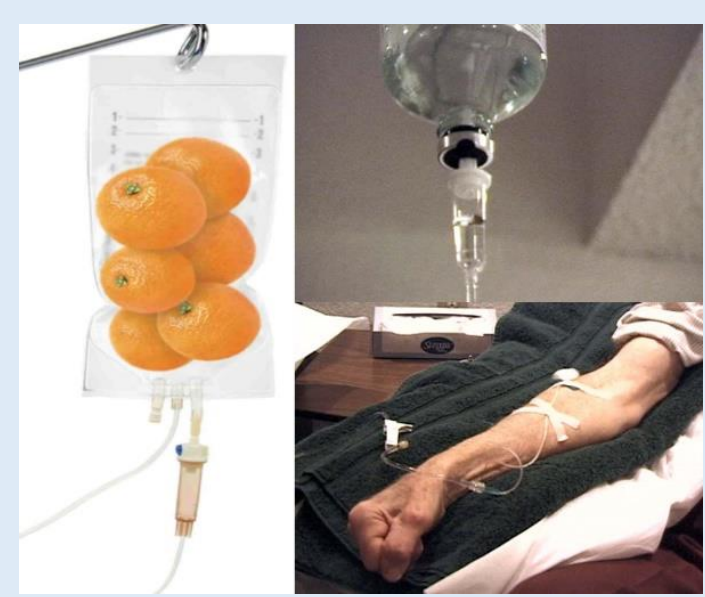
hematologic and blood chemistry parameters, and time to survival were monitored during treatment.

Subjects: 17 terminal colon cancer patients.

Outcome measures: Blood was collected to measure ascorbic acid (AA), absolute lymphocyte count (ALC), neutrophil to lymphocyte ratio (NLR), lactate dehydrogenase concentration (LDH), glucose concentration, ratio of immature neutrophils to total white blood cells (IN/WBC), albumin and creatinine concentrations. Patients' survival time was correlated with measured biomarkers.

Results: The evaluation of the initial blood chemistry parameters as prognostic factors of patients' survival demonstrated strong correlation with survival for lactate dehydrogenase, creatinine, and albumin levels. Continuous ascorbate infusions demonstrated a regulatory effect on ALC, lymphopenia, and NLR, which suggested a benefit of using medium continuous ascorbate doses for improvement of immune functioning. The rate of 
growth of LDH in patients with elevated initial levels was decreased in most cases. Treatments were accompanied by reduced serum glucose and uric acid concentrations. In addition, our data demonstrated that continuous IVC can be administered safely.

Conclusions: Continuous IVC infusions show potential to benefit colon cancer patients with minimal side effects. Further research and clinical studies investigating the efficacy of continuous IVC therapy for colon cancer are warranted.

Keywords: continuous infusion, ascorbic acid, colon cancer, survival, lymphopenia, lactate dehydrogenase, neutrophil to lymphocyte ratio.

CFFC 2020. This is an Open Access article distributed under the terms of the Creative Commons Attribution 4.0 License (http://creativecommons.org/licenses/by/4.0)

\section{INTRODUCTION}

Colorectal cancer is a major worldwide health problem that, despite recent improvements in treatment, continues to have a low response rate [1] It is a heterogeneous disease. Roughly forty percent of cases carry a KRAS oncogene mutation, which is associated with poor outcomes, poor overall survival, serious alterations in normal cell metabolism and resistance to chemotherapy $[2,3]$.

Intravenous ascorbic acid administration is being considered as a potential treatment for cancer [4]. Pharmacological concentrations of $A A$ induce antiproliferative, cytotoxic and genotoxic effects on colon cancer cells in vitro [5]. Tumors preferentially accumulate ascorbic acid, possibly by uptake via glucose transporters and by increased expression of AA transporters [6]. Ascorbic acid may interfere with glucose metabolism in colon cancer cells, which rely heavily on the Warburg effect to meet cellular energy needs by blocking essential enzymes or shutting down the pathway that allows KRAS mutationdependent enhancement of glucose absorption [7].

Other potential mechanisms of action for AA against colon cancer include upregulation of p53 and inhibition of hyaluronidase, an enzyme that aids tumor metastasis by destroying collagen [8,9]. Ascorbic acid is associated with the "normalization" of methylation markers ten-eleven translocation enzymes (TET) and 5-hydroxymethyl cytosine (5hmCyt), as well as with decreases in hypoxia inducible factor (HIF-1)-activated tumor growth $[10,11]$. In summary, ascorbic acid at pharmacological concentrations exerts a selectively cytotoxic effect toward tumors, by several potential mechanisms of action.

At the clinical level, AA is associated with improvement of quality-of-life in patients with advanced disease and improved tolerability of standard therapy [7]. In the present publication, we described previously unpublished parameters from the Riordan clinical study of the treatment of colon cancer patients by continuous ascorbic acid infusions, including blood chemistry and blood count parameters that are reportedly related to patient prognosis and degree of inflammation.

\section{MATERIAL AND METHODS}

Patient characteristics: Seventeen patients with late stage colon cancer were included in our study. $88 \%$ of the patients had a metastatic tumor. Written 
informed consent was provided by all patients included in this study. The ethics committees of the Eppley Institute for Research in Cancer and Allied Diseases at the University of Nebraska Medical Center (Omaha. NE) and the IRB of the Riordan Clinic approved the study.

Before participating in this study, all patients had undergone several chemotherapies, some with radiation and some without. A description of all recruited 24 patients and how the Phase 1 IVC continuous infusion clinical trial was conducted was previously given $[12,13]$. Since $70 \%$ of the participants in this study were patients with colon cancer, we decided to analyze this group separately.

For these subjects, information about the type of cancer, metastatic state, dosage during treatment, duration of the treatment, survival and adverse effects during intervention are presented in Table 1.

Table 1. Characteristics of colon cancer patients participating in a phase I clinical trial of continuous IVC infusions.

\begin{tabular}{|c|c|c|c|c|c|c|}
\hline Subject & Sex & Primary / Metastasis & $\begin{array}{c}\text { AA Dose } \\
\mathrm{mg} / \mathrm{kg} / \text { day }\end{array}$ & $\begin{array}{c}\text { Treatment } \\
\text { Time (week) }\end{array}$ & $\begin{array}{c}\text { Survival Time } \\
\text { (days) }\end{array}$ & Adverse effects \\
\hline 1 & M & Colon / Liver & 150 & 4 & 69 & ED \\
\hline 2 & $\mathrm{~F}$ & Colon / Liver, Lung & 710 & 8 & 456 & none \\
\hline 3 & $\mathrm{~F}$ & Colon / Liver & 290 & 8 & 143 & $\mathrm{DM}, \mathrm{NA}, \mathrm{F}$ \\
\hline 4 & M & Colon / Lung, Liver & 570 & 8 & 397 & none \\
\hline 5 & $\mathrm{~F}$ & Colon / Lung & 430 & 7 & 155 & ED, DM \\
\hline 6 & M & Colon / Liver & 290 & 2 & 36 & KS, DM \\
\hline 7 & $\mathrm{~F}$ & Colon / Lung & 430 & 3 & 21 & ED \\
\hline 8 & M & Colon / Liver & 430 & 8 & 80 & none \\
\hline 9 & $\mathrm{~F}$ & Colon / Liver & 150 & 8 & 67 & NA \\
\hline 10 & M & Colon / Liver & 430 & 48 & 334 & $\mathrm{SD}, \mathrm{NA}, \mathrm{K}$ \\
\hline 11 & $\mathrm{~F}$ & Colon & 430 & 6 & ND & NA \\
\hline 12 & M & Colon / Liver, Lung & 710 & 8 & 43 & $\mathrm{~F}, \mathrm{~K}$ \\
\hline 13 & M & Colon / Chest-Abd. Wall & 150 & 7 & 220 & DM \\
\hline 14 & $\mathrm{~F}$ & Colon / Liver & 290 & 6 & 142 & none \\
\hline 15 & M & Colon / Lung & 570 & 8 & ND & $E D, B P$ \\
\hline 16 & M & Colon / Liver & 710 & 8 & 110 & IWB \\
\hline 17 & M & Colon / Omentum & 290 & 8 & 173 & $A X$ \\
\hline
\end{tabular}

The abbreviations of side effects are: SD = Stable Disease, IWB = improved well-being, ED - edema, DM - dry mouth, NA - nausea, $\mathrm{BP}$-blood pressure, AX - anorexia, KS - kidney stones and K-hypokalemia. ND- not determined.

Intervention: Patients were divided into groups and given continuous infusions of $A A$ at specified doses: $150 \mathrm{mg} / \mathrm{kg} /$ day (3 patients), $290 \mathrm{mg} / \mathrm{kg} /$ day (4 patients), $430 \mathrm{mg} / \mathrm{kg} /$ day (5 patients), $510 \mathrm{mg} / \mathrm{kg} /$ day ( 2 patients) and $710 \mathrm{mg} / \mathrm{kg} /$ day (3 patients). To administer treatment, ascorbic acid was diluted in Lactated Ringers solution and administered continuously with a infusion pump (flow rates 20 $\mathrm{ml} /$ hour or $10 \mathrm{ml} /$ hour depending on the dose). Overall, fourteen of the patients received the treatment for at least six weeks. Subject \#10 showed stable disease and elected to continue ascorbic acid therapy for an additional forty-eight weeks. Patients' health, adverse events and tumor progression were 
monitored during treatment. Samples for routine blood chemistry were collected one week prior to therapy and weekly during treatment.

Statistical analysis: The inter-relationship between survival and blood parameters was examined using Spearman's correlation coefficients and regression analysis. Pairwise comparisons were analyzed by Wilcoxon Signed-Rank test. Two tailed $p$ values $<0.05$ were considered statistically significant. Statistical analysis was performed using Systat software (Version 13, Chicago, IL, USA).

\section{RESULTS}

Plasma ascorbate concentration: Pre-treatment plasma ascorbic acid concentrations were below normal in two-thirds of the subjects. Kinetic curves of $A A$ concentrations in blood during 21-28 days in the range of doses from lowest $150 \mathrm{mg} / \mathrm{kg} /$ day to highest $710 \mathrm{mg} / \mathrm{kg} /$ day, averaged for all patients in these dosage groups, are presented in Figure 1.

Plasma AA concentrations increased initially and then appeared to reach of steady state between 1.0 and $1.6 \mathrm{mM}$. The data for all four doses could be fitted to a single Michaelis-Menten type equation with a calculated maximum level of $1.19 \mathrm{mM}$ and a half-time to maximum of 0.87 days $(r=0.75)$. Concentrations in this range are shown to be necessary for anti-cancer effect [4,14]. Increasing dosages did not have a dramatic effect on the steady state AA concentrations, which may be explained by the saturation of tubular $\mathrm{AA}$ reabsorption and sodium-dependent transporters, leading the body to excrete AA more rapidly [15].

\section{Ascorbic acid in plasma}

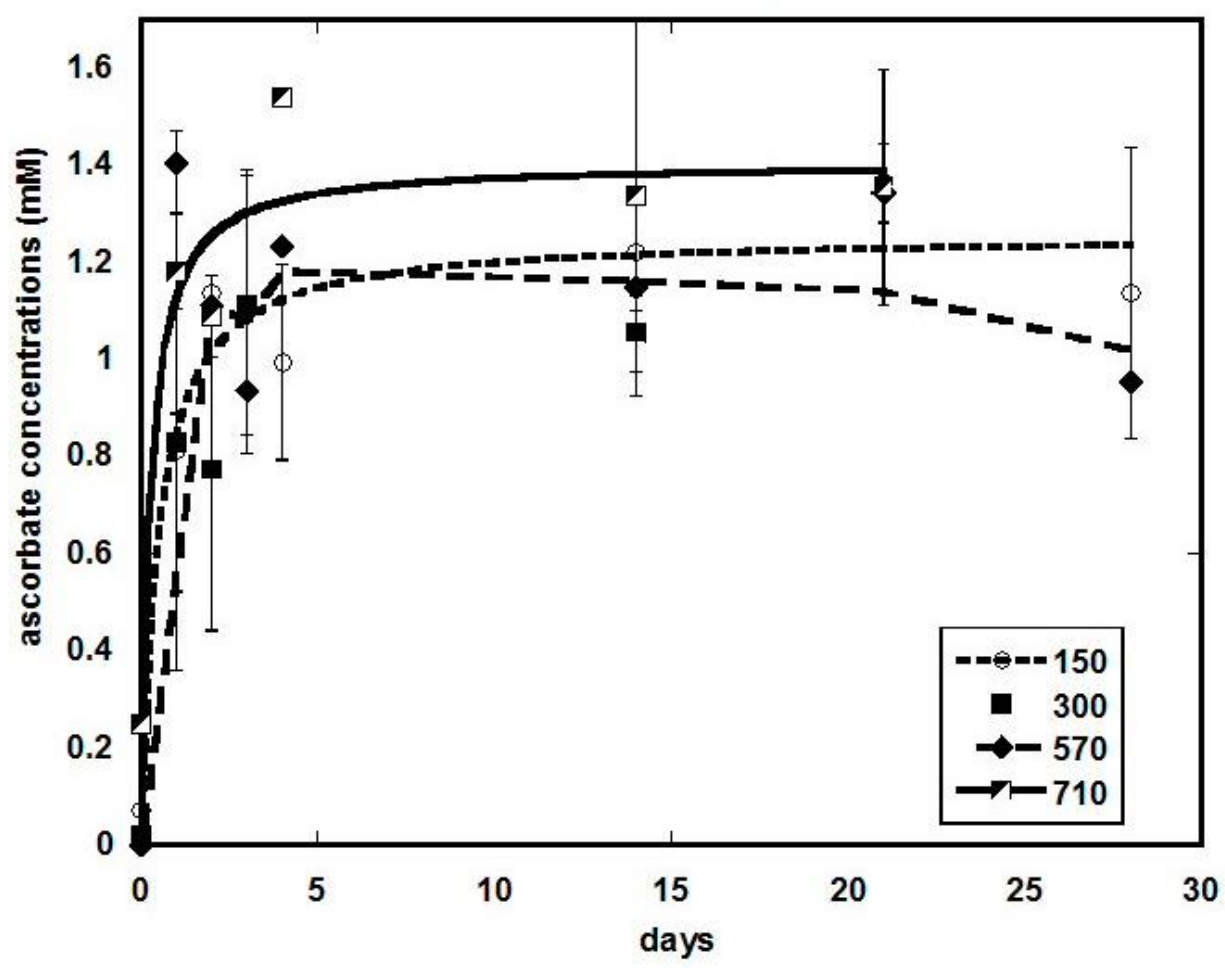

Figure 1: Average ascorbic acid concentrations (mM) for doses of $150 \mathrm{mg} / \mathrm{kg}, 290 \mathrm{mg} / \mathrm{kg}, 570 \mathrm{mg} / \mathrm{kg}$, and $710 \mathrm{mg} / \mathrm{kg}$ plotted against time 
Survival time and blood parameters: Table 2 shows how various blood counts and chemistry parameters measured prior to intravenous ascorbate therapy correlate with patient survival time. The Spearman correlation coefficients between survival and blood tests are presented in Table 2.

LDH showed a negative correlation with survival $\left(r_{s}=-0.78, p<0.04\right)$, while creatinine $\left(r_{s}=0.68, p<\right.$ $0.006)$ and albumin levels $\left(r_{s}=0.51, p<0.02\right)$ showed strong positive correlations with survival. There was weaker negative association between survival, NLR, immature neutrophils, glucose $\left(r_{s}\right.$ in range -0.33 to $0.39)$ and positive association with ALC $\left(r_{s}=0.31\right)$.

To demonstrate the prognostic value of these parameters, we compared survival times of subjects with normal parameter values to those with parameter values outside the normal range. Results are shown as box and whisker plots in Figure 2 .

For each of the parameters depicted, subjects with values in the normal range had significantly better survival rates than those with abnormal parameter values. Survival times were reduced for subjects with ALC below 1000 cells/L, NLR above 3.5, LDH above $250 \mathrm{U} / \mathrm{L}$, IN/WBC above 0.05, creatinine concentrations below $0.8 \mathrm{mg} / \mathrm{dL}$, and albumin levels below $3.5 \mathrm{~g} / \mathrm{dL}$. In addition, our data shows the cumulative effect of multiple abnormal parameter values. Survival time was lower for subjects with more abnormal values of these parameters.

Based on this analysis, we investigated the effect of the continuous IVC treatment on parameters correlated with survival.

Table 2. Correlation matrix between measured blood parameters and survival of the patients. Spearman correlation coefficients between patient survival time and parameters measured in colon cancer patients prior to treatment are indicated.

\begin{tabular}{|c|c|c|c|c|c|c|c|c|}
\hline & survival & ALC & NLR & LDH & glucose & IN/WBC & creatinine & uric acid \\
\hline survival & 1 & & & & & & & \\
\hline ALC & 0.31 & 1 & & & & & & \\
\hline NLR & -0.39 & -0.57 & 1 & & & & & \\
\hline LDH & -0.78 & -0.27 & 0.34 & 1 & & & & \\
\hline glucose & -0.37 & -0.42 & 0.40 & 0.13 & 1 & & & \\
\hline IN/WBC & -0.33 & -0.19 & 0.13 & 0.15 & 0.14 & 1 & & \\
\hline creatinine & 0.68 & -0.04 & -0.10 & -0.5 & -0.18 & -0.57 & 1 & \\
\hline uric acid & -0.21 & -0.01 & -0.13 & -0.11 & 0.01 & 0.07 & -0.12 & 1 \\
\hline A/G ratio & 0.46 & 0.25 & -0.30 & -0.12 & -0.03 & -0.26 & 0.45 & 0.06 \\
\hline albumin & 0.51 & 0.41 & -0.65 & -0.30 & -0.22 & -0.33 & 0.29 & 0.28 \\
\hline
\end{tabular}




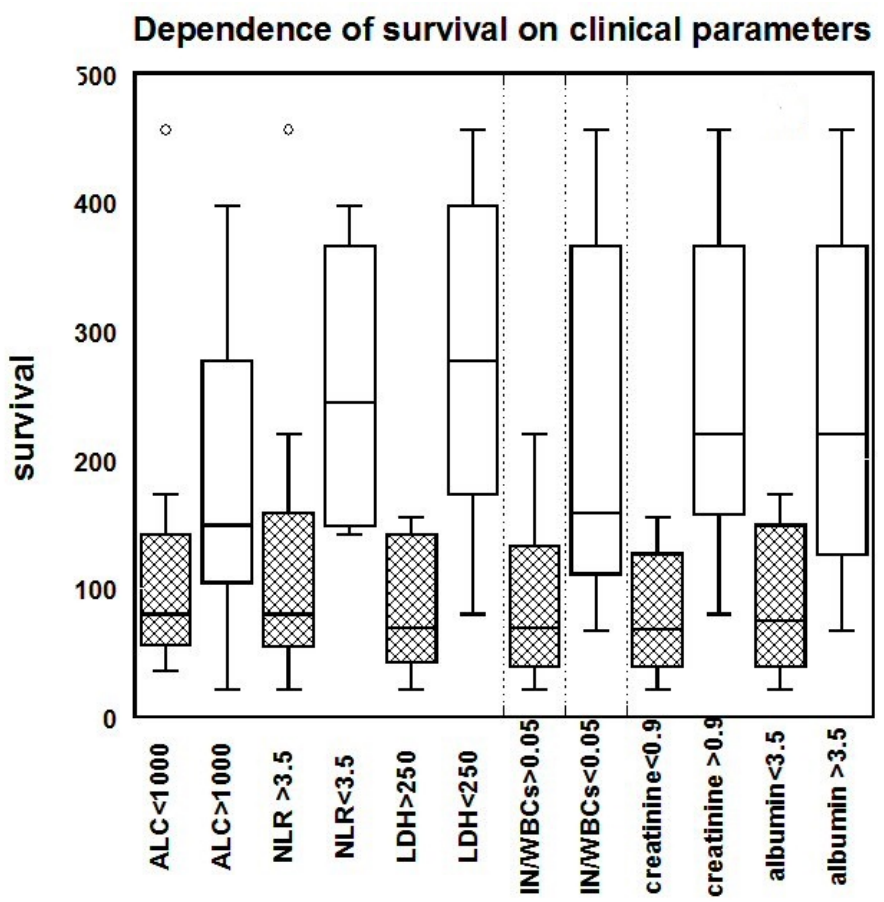

Figure 2. Survival time (days) of colon cancer patients with normal (clear boxes) or abnormal (shaded boxes) blood chemistry parameter values. Normal vs. Abnormal cutoffs: ALC $<1000$ cells/ul, NLR $<3.5$, LDH $<250$ U/L, IN/WBC $<5 \%$, creatinine $<0.8 \mathrm{mg} / \mathrm{dl}$, and albumin $<3.5 \mathrm{~g} / \mathrm{dl}$.

\section{Effect of continuous infusion on lymphocyte and} neutrophil counts: As lymphocytes and neutrophils have important roles in tumorigenesis and carcinogenesis, we tracked ALC and NLR during intravenous ascorbate infusions.

The effect of continuous infusions on the ALC was analyzed for all terminally ill colon cancer patients. Ten patients enrolled in the study had lymphopenia or ALC values below normal range (1300-4000 cells/ $\mu$ l) with severe lymphopenia (ALC < 1000 cells/ $\mu$ l) observed in seven subjects. Six of the seven with severe lymphopenia had improvement in ALC during ascorbate therapy (at an average increase of $53 \%$ ). Data for patients' lymphocytes one week before treatments, at the beginning of the treatment, and at the end of the treatment are shown in Table 3.

Distributions of the percent change of ALC and NLR before and after treatment are shown in Figure 3. The percentage of change in ALC was calculated based on the pre-treatment ALC, initial ALC values and the ALC at the end of the treatment.
Data in Figure 3 indicates that ALC values appear to drop in the week leading up to treatments, but then improve during the course of treatment. The average improvement during treatment was $28 \%$ (IQR: $-20 \%$ to $73 \%$ ) compared an average decrease of $-10 \%$ (IQR: $-25 \%$ to $13 \%$ ) in the week immediately before treatment.

In addition, we calculated the neutrophil-tolymphocyte ratios, as NLR is useful prognostic factors in a variety of cancers. Table 3 shows the NLRs for each of the seventeen subjects before and during treatment. For NLR, values appear to be rising in the week leading up to therapy, suggesting a poor prognosis for these subjects. Immediately prior to the onset of therapy, 12 subjects had above normal NLR levels (0.78 - 3.53). The improvement in the NLR during treatment is shown as down marks (DN) in the Table 3. Continuous ascorbate infusion resulted in improvement of NLR in seven of the 12 patients who showed abnormal NLR values initially. 
Table 3. Time course of ALC and NLR values in colon cancer patient given IVC infusions. $A L C_{p}=$ value one week pretreatment; $A L C_{0}=$ value at treatment start; $A L C_{F}=$ value at treatment end; $N L R_{p}=$ value one week pre-treatment; NLR $=$ value

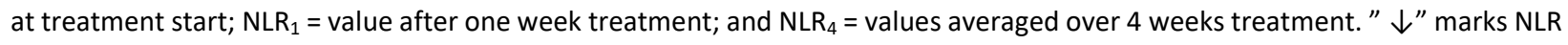
decrease during treatment.

\begin{tabular}{|c|c|c|c|c|c|c|c|c|}
\hline & $A L C_{p}$ & $\mathrm{ALC}_{0}$ & $\mathrm{ALC}_{\mathrm{F}}$ & NLR $_{\mathbf{P}}$ & $\mathbf{N L R}_{0}$ & $\mathrm{NLR}_{1}$ & $\mathrm{NLR}_{4}$ & Trend \\
\hline subject 1 & 1230 & 980 & 1056 & 4.73 & 5.64 & 5.00 & $6.22 \pm 0.87$ & \\
\hline subject 2 & 1512 & 972 & 1055 & 3.19 & 6.50 & 4.29 & $4.54 \pm 0.36$ & $\downarrow$ \\
\hline subject 3 & ND & 1261 & 755 & ND & 5.69 & 5.92 & $7.46 \pm 1.09$ & \\
\hline subject 4 & 1400 & 1590 & 1270.2 & 2.07 & 1.87 & 2.46 & $2.33 \pm 0.12$ & \\
\hline subject 5 & 2232 & 1971 & 1795.2 & 1.74 & 2.07 & 2.27 & $2.65 \pm 0.33$ & \\
\hline subject 6 & 420 & 518 & 897 & 13.67 & 12.29 & 6.23 & 6.23 & $\downarrow$ \\
\hline subject 7 & 1296 & 1560 & 1044 & 6.75 & 15.00 & 9.11 & ND & $\downarrow$ \\
\hline subject 8 & ND & 944 & 1751 & ND & 4.56 & ND & $5.86 \pm 0.55$ & \\
\hline subject 9 & 1850 & 1494 & 3843 & 2.48 & 3.89 & ND & $2.94 \pm 0.42$ & $\downarrow$ \\
\hline subject 10 & 4712 & 4712 & 7752 & 2.30 & 2.00 & 1.84 & $2.12 \pm 0.39$ & \\
\hline subject 11 & 2048 & 2205 & 1610 & 2.00 & 1.00 & 1.88 & $2.31 \pm 0.48$ & \\
\hline subject 12 & ND & 936 & 915 & ND & 3.67 & 2.33 & $3.86 \pm 0.89$ & \\
\hline subject 13 & 2162 & 1168 & 1584 & 2.74 & 4.31 & 2.95 & $2.71 \pm 0.40$ & $\downarrow$ \\
\hline subject 14 & 1045 & 1188 & 572.5 & 3.68 & 3.32 & 3.85 & $5.05 \pm 0.61$ & \\
\hline subject 15 & 1178 & 1311 & 1605 & 4.05 & 3.84 & 3.74 & $3.27 \pm 0.25$ & $\downarrow$ \\
\hline subject 16 & 496 & 624 & 1075.2 & ND & 3.84 & ND & 3.94 & \\
\hline subject 17 & 1170 & 902 & 2058 & 4.80 & 6.91 & 2.95 & $4.51 \pm 0.52$ & $\downarrow$ \\
\hline
\end{tabular}

\section{Change in ALC and NLR before and after treatment}

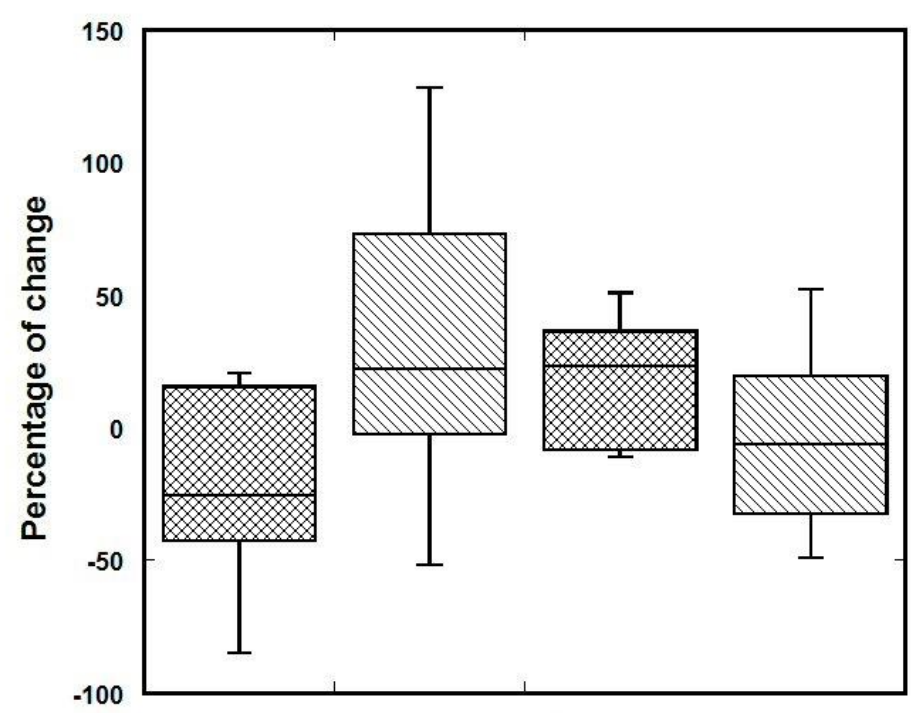

ALC before ALC post NLR before NLR after

Figure 3. Percent change in ALC and NLR before treatment compared to during IVC therapy in colon cancer patients who began treatment with abnormal values in each parameter. 
The percentage of NLR changes are shown in Figure 3 for subjects who started treatment with abnormal levels of each parameter. For these subjects, therapy tended to move parameter values in the direction for improving prognosis. These data suggest that continuous ascorbate treatment can result in the suppression or prevention of the progression of the rate of growth of NLR.

We also evaluated the change in the level of immature granulocytes during intervention. The ratio of immature neutrophils to total white blood cells (IN/WBC) was above normal (2\%) in ten patients, with the median IN/WBC in these subjects being 7.5\% (IQR $=4.9 \%$ to $12.6 \%$ ). Treatment reduced these values to a median of $3.4 \%$ (IQR $=0.4 \%$ to $4.6 \%$ ).

\section{Effect of ascorbic acid on blood chemistry} parameters: LDH is elevated in many types of cancers; it has been linked to tumor growth, maintenance, and invasion [16]. Regression analysis (Table 2) demonstrated a significant correlation between colon cancer patient survival time and LDH $(P<0.004)$. Subjects with $L D H$ values within the normal range (140 to $280 \mathrm{U} / \mathrm{L}$ ) had a median survival time of 277 days (IQR $=150$ to 412 ) while subjects with elevated LDH values had a median survival time of 90 days (IQR $=44$ to 143$)$. LDH concentrations before IVC therapy were above the normal range in $56 \%$ of the patients. Overall, nine subjects showed continued increases in LDH while six showed an LDH decrease.

Hyperglycemia is common in cancer patients [17]. Sixty percent of the colon cancer patients in our study had above normal blood glucose concentrations $(>100 \mathrm{mg} / \mathrm{dl})$. The effect of IVC on plasma glucose concentration is shown in Figure 4, where the average percentage of change in glucose concentration from the pre-treatment value is plotted against the initial glucose concentration.
For patients with above-normal pre-treatment glucose concentrations, plasma glucose concentrations decreased by an average of $21 \%$ (IQR $=-31 \%$ to $+13 \%$ ) during treatment. The most dramatic decreases in glucose levels occurred when the initial glucose concentrations were at their highest levels.

Continuous ascorbate treatments corresponded to significant reductions in serum uric acid, noticeable as early as the first week after treatment. Recent evidence has demonstrated that hyperuricemia is associated with excess cancer risk, recurrence, and mortality [18]. During treatment, all patients experienced a significant uric acid lowering effect. The median values of UA were $5.1 \mathrm{mg} / \mathrm{dL}(I Q R=4.3$ to 5.7 ) before treatment and $2.2 \mathrm{mg} / \mathrm{dL}$ (IQR $=1.4$ to 2.7) after one week of treatment. The reduction in UA after one week of treatment depended on ascorbate dosage.

Two other blood chemistry parameters that correlate with survival in our study were serum albumin and creatinine. Our data demonstrated high correlations between carnitine and albumin levels and survival colon cancer patients $(r=0.68$ and $r=0.51)$. The correlation of initial (pre-treatment) creatinine and albumin levels with survival time is shown in Figure 5(a, b).

Albumin was below normal range (3.5 to 5.0 $\mathrm{g} / \mathrm{dL}$ ) in 7 patients (median $=3.3 \mathrm{~g} / \mathrm{dL}, \mathrm{IQR}=3.2$ to 3.4). As it was shown in Table 2 and Figure 2, albumin has a prognostic value in our colorectal cancer patients. Albumin levels were decreasing in most of these subjects prior to therapy and during therapy, and we do not have evidence that treatment affected albumin levels in this study.

The average decrease in creatinine during treatment was $14 \%(I Q R=-4 \%$ to $-24 \%)$, a statistically significant change $(p<0.001)$. Thus, intervention did not prevent decreases in creatinine during this study. 


\section{Change in glucose concentration during therapy}

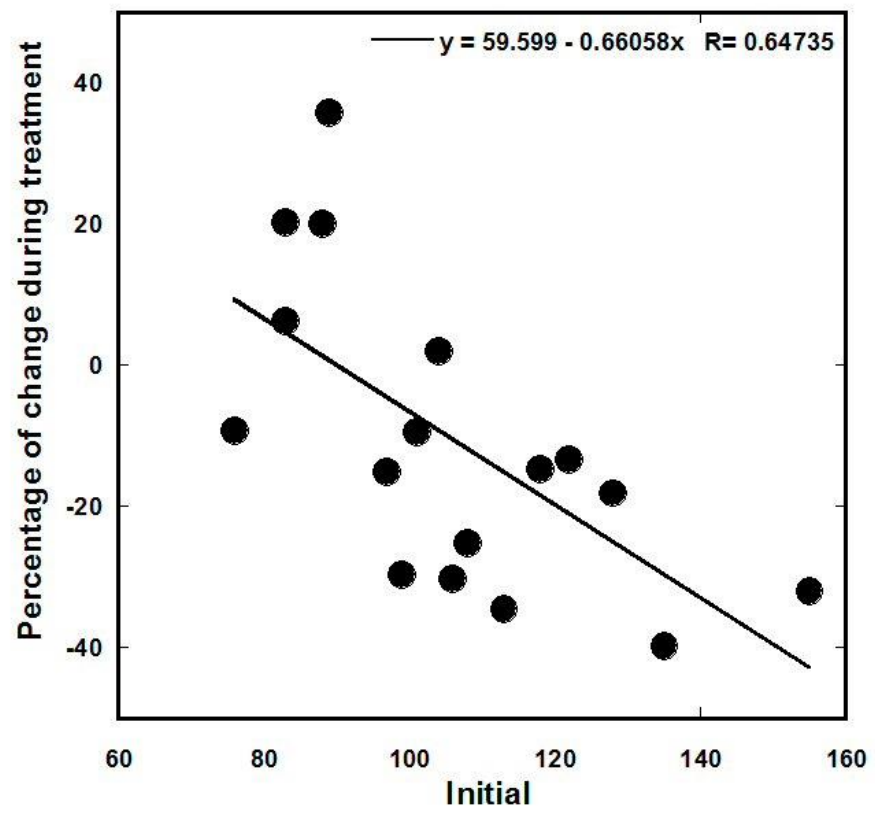

Figure 4. Percentage of plasma glucose concentration change during IVC therapy as function of pre-treatment glucose concentrations $(\mathrm{mg} / \mathrm{dL})$. The line represents the linear extrapolation of the data.
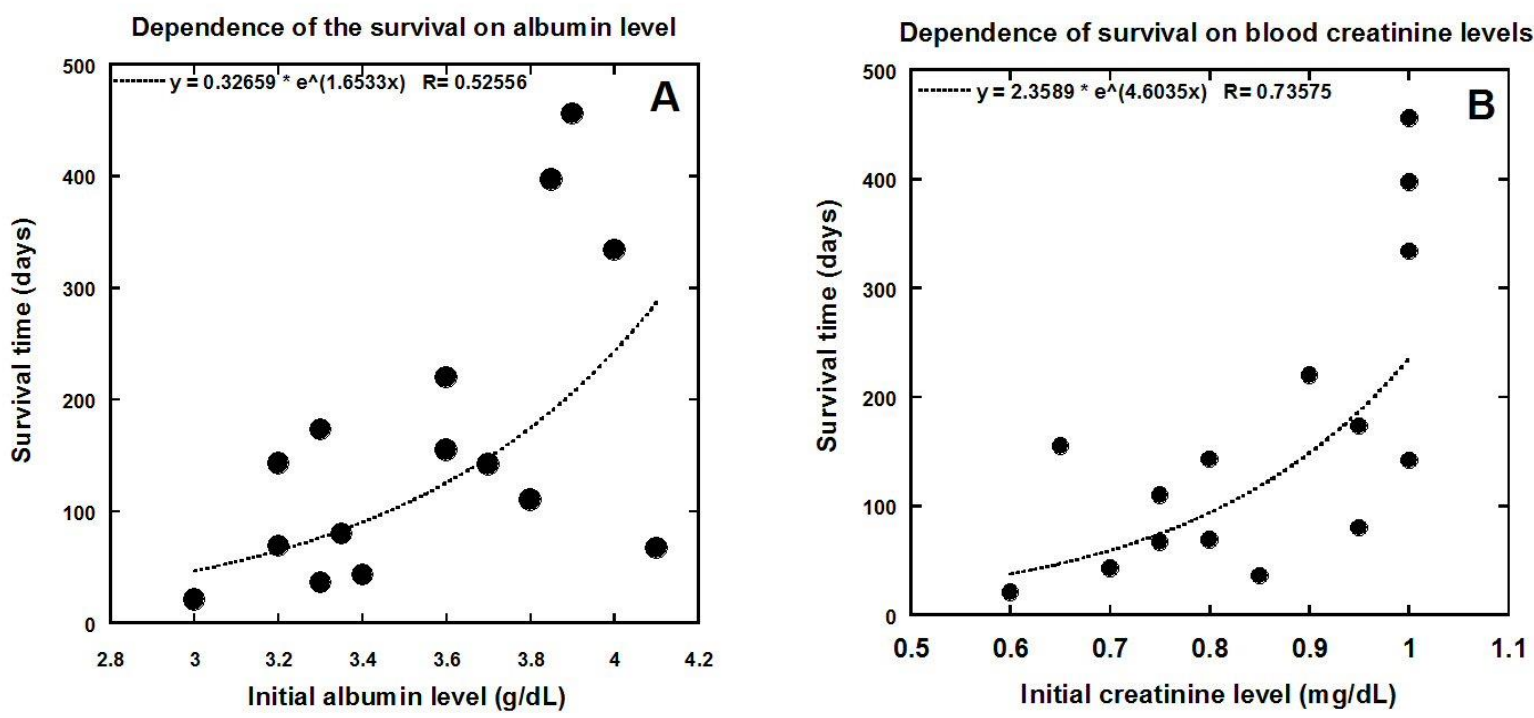

Figure $5(\mathbf{a}, \mathbf{b})$. Correlation of colon cancer patient survival with initial levels of albumin and creatinine.

\section{DISCUSSION}

We analyzed data of seventeen colon cancer patients from the Phase I clinical trial given continuous IVC infusions [12]. The eight-week trial involved terminal patients with poor prognosis. Considering recent interest in potential biological effects of vitamin $\mathrm{C}$ on cancer survival and improvements in quality of life, we analyzed previously unpublished data from this study in the subgroup of colon cancer patients. Our purpose was two-fold: to see if these parameters served as prognostic indicators of survival for colon cancer patients in the study and to determine if there 
were any improvements in parameter values for these subjects during therapy.

The continuous infusions of ascorbate at doses used in this pilot clinical trial lead to sustained plasma AA concentrations (1.0 to $1.6 \mathrm{mM}$ ) that, based on experimental evidence, are sufficient for anti-tumor effects.

In the present study, we evaluated the initial blood chemistry parameters as prognostic factors of patients' survival. According to our data, a strong correlation with survival was found for $\mathrm{LDH}$, creatinine and albumin levels, and survival time was lower for subjects with abnormal values of the measured parameters.

Serum albumin level has been found to be an independent prognostic factor for survival in various cancers including colorectal cancer [19]. In our group of patients, seven patients had level of albumin lower than normal range with tendency to decrease on average on $8.3 \%$ during intervention.

Creatinine was found to be another prognostic parameter of survival for late stage colon cancer patients.

Another potential biomarker of patients' survival was the level of lactate dehydrogenase ( $r=-$ 0.78). Only six patients showed the decrease in the level of LDH during treatment; however, the rate of growth of this parameter in patients with LDH higher than normal range was suppressed in most of patients.

Analysis of white blood cell counts for colon cancer patients in our trial provided two main observations: patient lymphocytes tended to decrease with time prior to therapy; and patients who had below normal lymphocyte counts at the onset of therapy tended to see an increase in lymphocyte numbers during treatment. The present analysis demonstrated the regulatory effect of continuous ascorbate on ALC and lymphopenia and suggested a strategic benefit of using medium ascorbate doses in continuous infusions for improvement immune cell counts.

Analysis of neutrophil-to-lymphocyte ratios also demonstrated a potentially beneficial regulatory effect of treatment on the immune cell population. In the present study, most of the patients entered the trial with above-normal NLRs. Continuous ascorbate therapy decreased NLR in 7 of 12 patients who had initially above-normal NLR levels.

Our analysis also demonstrated an effect of treatment on immature granulocytes. High proportion of immature neutrophils predicts infection [20]. In our study, $60 \%$ of the patients had elevated IN/WBCs (averaged for the period before treatment) with a median of $7.5 \%$. During treatment, the median IN/WBCs for these patients decreased to $3.35 \%$. This may indicate a decrease in infection and/or inflammation level in these patients during therapy.

In the present study, continuous ascorbate therapy was associated with decreases in plasma glucose concentrations in patients with hyperglycemia and a reduction in plasma uric acid.

Our data confirmed that continuous IVC can be administered safely. For prevention of hypokalemia, the level of electrolytes should be controlled during treatment and include supplementation. In addition, renal function should be monitored, and patients with the history of kidney disease are not recommended for such treatments. Adverse events were more frequent at the higher IVC doses used [13], and based on the pharmacokinetic data, we can 
suggest that higher doses in continuous infusion ascorbic acid therapy may result in more side effects without significant increasing plasma ascorbate concentrations and improvement biomarkers beyond those obtained at lower doses.

This analysis, to our knowledge, is the first clinical study of continuous ascorbate infusion that includes analysis of biological markers correlating with survival and monitors how these markers change during therapy. We conclude that injected doses between $10-20 \mathrm{~g} /$ day are enough to achieve plasma ascorbate concentrations of $1 \mathrm{mM}$ or higher. This therapy also showed signs of improving blood cell count and chemistry parameters that are prognostic indicators of patient survival.

The weaknesses of the study were low number of patients, late stage of cancer (untreatable, metastatic colon cancer), the lack of a control group, and the likelihood that subjects had immune systems that were damaged by prior treatments such as chemotherapy and irradiation. Future studies should be designed to address these issues.

\section{CONCLUSIONS}

In conclusion, the analysis of the parameters that served as prognostic indicators of colon cancer patients' survival and evaluation of the improvements of described parameters' values for these subjects during therapy demonstrated that continuous ascorbate infusions benefit colon cancer patients. The data suggested a regimen of continuous infusions for improving immune functioning and cancer biomarkers. In addition, our analysis demonstrated that continuous ascorbate can be administered safely.

Abbreviations: AA: ascorbic acid; LDH: lactate dehydrogenase; ALC: absolute lymphocyte counts;
NLR: neutrophil to lymphocyte ratio; IN/WBC: the ratio of immature neutrophils to white blood cells; A:G: albumin to globulin ratio; UA: uric acid.

Disclosure Statement: No competing financial interests exist.

Author' contributions: NM analyzed data, made the statistical calculations, and prepared the manuscript.

Funding: This research received no external funding.

\section{REFERENCES}

1. Allen WL, Coyle VM, Johnston PG. Predicting the outcome of chemotherapy for colorectal cancer. Curr. Opin 2006; 6: 332-336.

2. Tan $\mathrm{C}, \mathrm{Du} \mathrm{X}$. Kras mutation testing in metastatic colorectal cancer. World J. Gastroenterol 2012; 18: 5171.

3. Lievre A, Bachet JB, Le Corre D, et al. Kras mutation status is predictive of response to cetuximab therapy in colorectal cancer. Cancer Res 2006; 66: 3992-3995.

4. Dua J, Cullena JJ, Buettnera GR. Ascorbic acid: Chemistry, biology and the treatment of cancer. Biochim Biophys Acta $2012 ; 1826:$ 443-457.

5. Pires AS, Marques CR, Encarnação JC, Abrantes AM, Mamede AC, Laranjo M, Gonçalves AC, Sarmento-Ribeiro $A B$, Botelho MF. Ascorbic acid and colon cancer: an oxidative stimulus to cell death depending on cell profile. Eur J cell Bio. 2016; 95: 208-218.

6. Casciari J, Riordan H, Miranfa-Massari J, Gonzalez M. Effects of high dose ascorbate administration on L-10 tumor growth in guinea pigs. Puero Rico Health Sciences Journal 2005; 24(2):145-150

7. Halabi I, Bejjany R, Nasr R, et al. Ascorbic acid in colon cancer : from the basic to the clinical applications. Int. J. Mol. Sci 2018; 19: 2752.

8. An SH, Kang JH, Kim DH, Lee MS. Vitamin C increases the apoptosis via up-regulation p53 during cisplatin treatment in human colon cancer cell. BMB reports 2011; 44. 3. 211.

9. Shi Y. Histone lysine demethylases: Emerging roles in development, physiology and disease. Nat Rev Genet 2007; 8: 829-833.

10. Minor EA, Court BL, Young JI, Wang G. Ascorbate induces ten-eleven translocation (Tet) methylcytosine dioxygenase-mediated generation of 5- 
hydroxymethylcytosine. J Biol Chem 2013; 288: 1366974.

11. Kuiper C, Dachs GU, Currie MJ, Vissers MC. Intracellular ascorbate enhances hypoxia-inducible factor (hif)hydroxylase activity and preferentially suppresses the hif1 transcriptional response. Free Radic. Biol. Med 2014; 69: $308-317$

12. Riordan HD, Casciari JJ, Gonzalez MJ, et al. A pilot clinical study of continuous intravenous ascorbate in terminal cancer patients. P R Health Sci J 2005; 24: 269-276.

13. Mikirova N, Casciari J, Hunninghake R. Continuous intravenous vitamin $\mathrm{C}$ in the cancer treatment: reevaluation of a Phase I clinical study. Functional Foods in Health and Disease 2019; 9(3): 180-204.

14. Campbell EJ, Vissers MCM, Wohlrab C, et al. Pharmacokinetic and anti-cancer properties of high dose ascorbate in solid tumors of ascorbate-dependent mice. Free Radical Biology and Medicine 2016; 99: 151-462.
15. Blanchard J, Tozer TN, Rowland M. Pharmacokinetic perspectives on mega doses of ascorbic acid. Am J Clin Nutr 1997; 66: 1165-71.

16. Suh SY, Ahn HY. Lactate dehydrogenase as a prognostic factor for survival time of terminally ill cancer patients: a preliminary study. Eur. J Cancer 2007; 43: 1051-1059.

17. Ryu TY, Jiyoung Park J, Scherer PE. Hyperglycemia as a Risk Factor for Cancer Progression. Diabetes Metab J $2014 ; 38: 330-336$

18. Strasak AM, Rapp K, Hilbe W, et al. Serum uric acid and risk of cancer mortality in a large prospective male cohort. Cancer Causes Control 2007; 18(9):1021-9.

19. Heys SD, Walker LG, Deehan DJ, Eremin OE. Serum albumin: a prognostic indicator in patients with colorectal cancer. J R Coll Surg Edinb 1998; 43: 163-168

20. van der Geest PJ, Mohseni M, Brouwer R, et al. Immature granulocytes predict microbial infection and its adverse sequelae in the intensive care unit. Journal of critical care 2014; 29: 523-527. 